\title{
An Extended Generalized Lindley Distribution and Its Applications to Lifetime Data
}

\author{
H. Torabi*, M. Falahati-Naeini and N.H. Montazeri \\ Yazd University
}

\begin{abstract}
In this paper, a four parameters extension of the generalized Lindley distribution is introduced. The new distribution includes the power Lindley, Lindley, generalized (Stacy) gamma, gamma, Weibull, Rayleigh, exponential and half-normal distribution. Several statistical properties of the distribution are explored. Then, a bivariate version of the proposed distribution is derived. Using a simulation study, some estimation methods for the parameters of the distribution are compared. Finally, a real data application illustrates the performance of our proposed distribution.
\end{abstract}

Keywords. AIC; BIC; CAIC; generalized Lindley distribution; generalized (Stacy) gamma distribution; HQIC; minimum spacing distance estimator; power Lindley distribution.

MSC 2010: 62F10, 62N05, 60E10, 60E15.

\section{Introduction}

The Lindley distribution, with probability density function (pdf)

$$
f(x ; \theta)=\frac{\theta^{2}(1+x) e^{-\theta x}}{1+\theta}, \quad x>0, \quad \theta>0,
$$

was introduced by Lindley (1958). Ghitany et al. (2008) investigated Lindley distribution in the context of reliability analysis.

* Corresponding author 
Seven generalization of the Lindley distribution are proposed by some authors as follows:

- Zakerzadeh and Dolati (2009) introduced a generalized Lindley distribution with the pdf

$$
f(x ; \alpha, \theta, \gamma)=\frac{\theta^{\alpha+1} x^{\alpha-1}(\alpha+\gamma x) e^{-\theta x}}{(\theta+\gamma) \Gamma(\alpha+1)}, \quad x>0, \quad \alpha, \theta, \gamma>0 .
$$

The distribution in the case $\alpha=\gamma=1$ is reduced to the Lindley distribution.

- Ghitany et al. (2011) introduced the weighted Lindley distribution with the pdf

$$
f(x ; \alpha, \theta)=\frac{\theta^{\alpha+1}}{(\theta+\alpha) \Gamma(\alpha)} x^{\alpha-1}(1+x) e^{-\theta x}, \quad x>0, \quad \alpha, \theta>0,
$$

which the Lindley distribution is obtained if $\alpha=1$.

- Ghitany et al. (2012) investigate the Marshall-Olkin extended Lindley distribution with the pdf

$$
\begin{aligned}
& f(x ; \alpha, \theta)=\frac{\alpha \theta^{2}(1+\theta)(1+x) e^{-\theta x}}{\left\{(1+\theta)-(1-\alpha)(1+\theta+\theta x) e^{-\theta x}\right\}^{2}}, \\
& \quad x>0, \quad 0<\alpha \leqslant 1, \theta>0 .
\end{aligned}
$$

Note the distribution in the special case $\alpha=1$ is reduced to the Lindley distribution.

- Shanker and Mishra (2013) introduced the quasi Lindley distribution with the pdf

$$
f(x ; \alpha, \theta)=\frac{\theta(\alpha+x \theta)}{\alpha+1} e^{-\theta x}, \quad x>0, \quad \alpha>-1, \theta>0 .
$$

This distribution in the case $\alpha=\theta$, is changed to the Lindley distribution.

- Ghitany et al. (2013) introduced the power Lindley distribution with the pdf

$$
f(x ; \theta, \beta)=\frac{\beta \theta^{2}}{\theta+1}\left(1+x^{\beta}\right) x^{\beta-1} \exp \left\{-\theta x^{\beta}\right\}, \quad x>0, \theta, \beta>0,
$$

were in the special case $\beta=1$ is reduced to the Lindley distribution. 
- Warahena-Liyanage and Pararai (2014) introduced the exponentiated power Lindley distribution with the pdf

$$
\begin{gathered}
f(x ; \beta, \theta, w)=\frac{\beta \theta^{2} w\left(1+x^{\beta}\right) x^{\beta-1} e^{-\theta x^{\beta}}}{(\theta+1)}\left\{1-\left(1+\frac{\theta x^{\beta}}{\theta+1}\right) e^{-\theta x^{\beta}}\right\}^{w-1}, \\
x>0, \quad \alpha, \theta, w>0,
\end{gathered}
$$

were in the special case $\beta=w=1$ is reduced to the Lindley distribution.

- Pararai et. al. (2015) introduced the beta exponentiated power Lindley distribution with the pdf

$$
\begin{aligned}
& f(x ; \beta, \theta, w, a, b)=\frac{\beta \theta^{2} w\left(1+x^{\beta}\right) x^{\beta-1} e^{-\theta x^{\beta}}}{(\theta+1)}\left\{1-\left(1+\frac{\theta x^{\beta}}{\theta+1}\right) e^{-\theta x^{\beta}}\right\}^{w a-1} \\
& \times\left[1-\left\{1-\left(1+\frac{\theta x^{\beta}}{\theta+1}\right) e^{-\theta x^{\beta}}\right\}^{w}\right]^{b-1}, \quad x>0, \quad \alpha, \theta, w, a, b>0
\end{aligned}
$$

were in the special case $\beta=w=a=b=1$ is reduced to the Lindley distribution.

The current article offers a distribution which generalizes the generalized Lindley distribution (2) and it is based on a certain mixture of two generalized gamma (Stacy gamma) distributions.

The purpose of this paper is to develop a four-parameter alternative to several lifetime distributions including the Lindly, genearlized Lindly, power-Lindly, generalized gamma, gamma, Weibull, exponentil, exponentiated Lindley, Rayleigh and half-normal distributions. In this context, we propose and develop the statistical properties of the Extended Generalized Lindley (EGL) distribution and show that it is a far better model for reliability analysis. Finally, applications of the model to real data sets in order to illustrate the applicability and usefulness of the EGL distribution are presented.

The paper is organized as follows:

Section 2 introduces an extended generalized Lindley distribution and presents its basic properties including the behaviour of the density and hazard rate functions, and some results on stochastic orderings. The moments of EGL distribution, the moment generating function (mgf) and the characteristic 
function are derived in Section 3. Mean residual function and scaled total time are obtained in Section 4 together with Lorenz curve and Bonferroni curve which are obtained in Section 5. Section 6 discusses a bivariate derivation of the proposed model and then the estimation of parameters is discussed in Section 7. In Section 8, we present the simulation issues of the extended generalized Lindley distribution. An application of the EGL distribution and comparing with other distributions, are given in Section 9.

\section{Definition and Some Properties}

In this section, we introduce an extended generalized Lindley distribution and study its basic properties.

Assume

$$
f_{g g}(x ; \alpha, \beta, \theta)=\frac{\beta \theta^{\alpha} x^{\alpha \beta-1} e^{-\theta x^{\beta}}}{\Gamma(\alpha)}, \quad x>0, \quad \alpha, \beta, \theta>0,
$$

is the density function of the generalized gamma (Stacy gamma) distribution, denoted by $\operatorname{GG}(\alpha, \beta, \theta)$. Let $V_{1}$ and $V_{2}$ be two independent random variables distributed according to $\operatorname{GG}(\alpha, \beta, \theta)$ and $\operatorname{GG}(\alpha+1, \beta, \theta)$, respectively. For $\gamma \geqslant 0$, consider the random variable $X=V_{1}$ with probability $\frac{\theta}{\theta+\gamma}$, and $X=V_{2}$ with probability $\frac{\gamma}{\theta+\gamma}$. Then density function of $X$ is as follows $f(x ; \alpha, \beta, \theta, \gamma)=\frac{e^{-\theta x^{\beta}} \beta\left\{\alpha x^{\alpha \beta}+\gamma x^{(\alpha+1) \beta}\right\} \theta^{\alpha+1}}{x(\gamma+\theta) \Gamma(\alpha+1)}, \quad x>0, \alpha, \beta, \theta, \gamma>0 .(10)$

We say that the random variable $X$ has an extended generalized Lindley (EGL) distribution, if $X$ has the density function defined by (10) and denote the extended generalized Lindley distribution with the parameters $\alpha, \beta, \theta$ and $\gamma$ by $\operatorname{EGL}(\alpha, \beta, \theta, \gamma)$. Note that if $Y$ has the generalized Lindley distribution, then $X=Y^{1 / \beta}$ has $\operatorname{EGL}(\alpha, \beta, \theta, \gamma)$; hence the EGL distribution can be consider as a power generalized Lindley distribution.

\subsection{Special Cases of the EGL Distribution}

The EGL distribution contains a large number of distributions. Here, we consider some special cases:

(a) For $\beta=1$, the EGL distribution reduces to the generalized Lindley distribution (2) with parameters $\alpha, \theta$ and $\gamma$; 
(b) For $\alpha=\gamma=1$, the EGL distribution reduces to the power Lindley distribution with parameter $\theta$ and $\beta$, Equation (6);

(c) For $\alpha=\beta=\gamma=1$, the EGL distribution reduces to the density function of the Lindley distribution with parameters $\theta$, Equation (1);

(d) For $\gamma=0$, the EGL distribution reduces to the generalized gamma (Stacy gamma) distribution with parameters $\alpha, \beta$ and $\theta$, Equation (9);

(e) For $\gamma=0$ and $\beta=1$, the EGL distribution reduces to the gamma distribution with parameters $\alpha$ and $\theta$;

$$
f(x ; \alpha, \theta)=\frac{\theta^{\alpha} x^{\alpha-1} e^{-\theta x}}{\Gamma(\alpha)},
$$

(f) For $\alpha=1, \gamma=0$, the EGL distribution reduces to the Weibull distribution with parameters $\beta$ and $\theta$;

$$
f(x ; \beta, \theta)=\beta \theta x^{\beta-1} e^{-\theta x^{\beta}}, \quad x>0,
$$

(g) For $\alpha=1, \beta=2, \gamma=0$, the EGL distribution reduces to the Rayleigh distribution with parameter $\theta$;

$$
f(x ; \theta)=2 \theta x e^{-\theta x^{2}}, \quad x>0,
$$

(h) For $\alpha=\beta=1$ and $\gamma=0$, the EGL distribution reduces to the exponential distribution;

$$
f(x ; \theta)=\theta e^{-\theta x}, \quad x>0,
$$

(i) For $\alpha=\frac{1}{2}, \beta=2, \gamma=0$, the EGL distribution reduces to the halfnormal distribution with parameter $\frac{1}{\sqrt{2 \theta}}$;

$$
f(x ; \theta)=\frac{2 \sqrt{\theta}}{\sqrt{\pi}} \exp \left\{-\theta x^{2}\right\}, \quad x>0,
$$



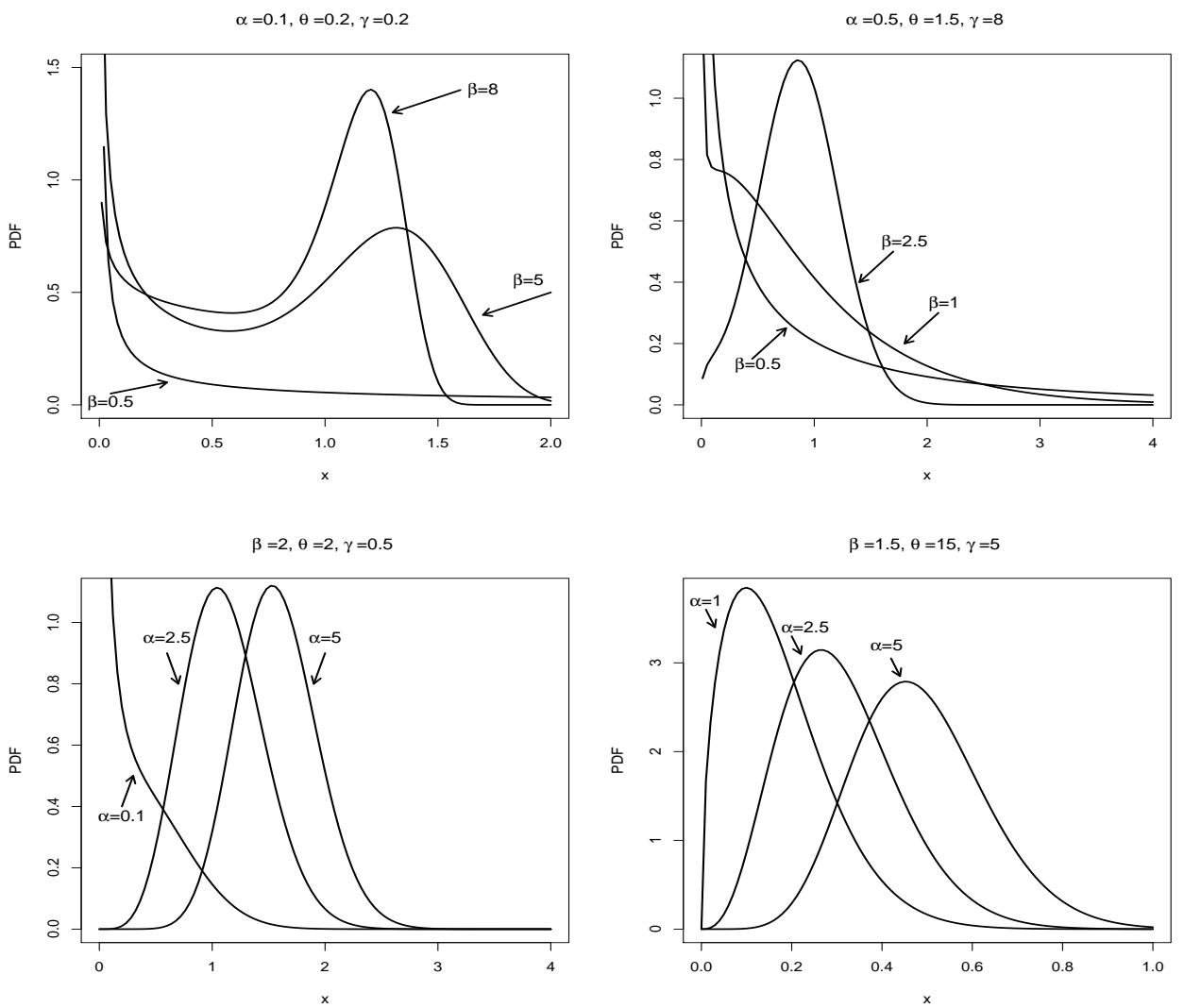

Figure 1. Plot of the pdf of the EGL distribution for some selected parameters $\alpha, \beta, \theta$ and $\gamma$.

\subsection{Shape}

Figure 1 shows the pdf of the EGL distribution for some selected parameters $\alpha, \beta, \theta$ and $\gamma$. This figure shows that the EGL distribution can be used for left-skewed, right-skewed and symmetric data.

Let $h(x)=\frac{f(x)}{1-F(x)}$ be the hazard rate function of the random variable $X$ distributed according to $\operatorname{EGL}(\alpha, \beta, \theta, \gamma)$. Then $h(x)$ has different behaviour depending on its parameters.

Figure 2 shows the hazard rate function of the EGL distribution for some selected parameters $\alpha, \beta, \theta$ and $\gamma$. 

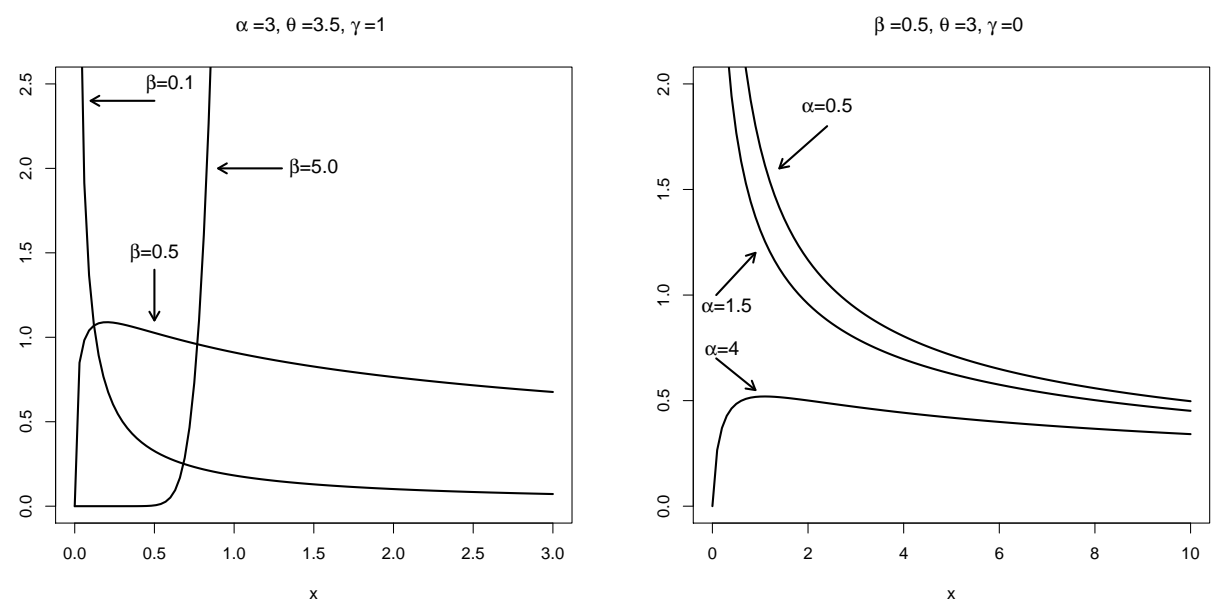

Figure 2. Plot of the hazard function of the EGL distribution for some selected parameters $\alpha, \beta, \theta$ and $\gamma$.

\subsection{Stochastic Orders}

A random variable $X$ is said to be stochastically smaller than $Y$ (denoted by $X \prec_{s} Y$ ), if $F_{X}(t) \geqslant F_{Y}(t)$ for all $t$.

Two stronger criterions are the hazard rate order (denoted by $X \prec_{h r}$ Y) if $h_{X}(t) \geqslant h_{Y}(t)$, for all $t$, and the likelihood ratio order (denoted by $\left.X \prec_{l r} Y\right)$, if $f_{X}(t) / f_{Y}(t)$ is decreasing in $t$. Note that

$$
X \prec_{l r} Y \Longrightarrow X \prec_{h r} Y \Longrightarrow X \prec_{s} Y ;
$$

for details of the proof, see Shaked and Shanthikumar (1994).

Let $X_{i}$ be a random variable distributed according to (10) with the parameters $\left(\alpha_{i}, \beta_{i}, \theta_{i}, \gamma_{i}\right)$, for $\mathrm{i}=1,2$. Then

$$
\begin{aligned}
\frac{d}{d y} \log \left\{\frac{f_{X_{1}}(x)}{f_{X_{2}}(x)}\right\}= & \frac{d}{d x}\left[\log f_{X_{1}}(x)-\log f_{X_{2}}(x)\right] \\
= & \frac{\beta_{1} \alpha_{1}-\beta_{2} \alpha_{2}}{x}+\frac{\beta_{2} \theta_{2} x^{\beta_{2}}-\beta_{1} \theta_{1} x^{\beta_{1}}}{x} \\
& +\frac{\beta_{1} \gamma_{1} x^{\beta_{1}-1}}{\left(\alpha_{1}+\gamma_{1} x^{\beta_{1}}\right)}-\frac{\beta_{2} \gamma_{2} x^{\beta_{2}-1}}{\left(\alpha_{2}+\gamma_{2} x^{\beta_{2}}\right)} .
\end{aligned}
$$


Clearly, if $\alpha_{1}=\alpha_{2}$ and $\beta_{1}=\beta_{2}$, then (11) is negative when $\theta_{1} \geqslant \theta_{2}$ and $\gamma_{1} \leqslant \gamma_{2}$.

When $\theta_{1}=\theta_{2}, \gamma_{1}=\gamma_{2}, \beta_{1}=\beta_{2}$, it can be shown that the expression (11) is negative for $\alpha_{1} \leqslant \alpha_{2}$.

Theorem 1. Let $X_{1}$ and $X_{2}$ be two random variables having the EGL distribution with the parameters $\left(\alpha_{i}, \beta_{i}, \theta_{i}, \gamma_{i}\right), i=1$, 2. Then the followings hold

i) If $\theta_{1}=\theta_{2}, \gamma_{1}=\gamma_{2}, \beta_{1}=\beta_{2}$ and $\alpha_{1} \leqslant \alpha_{2}$, then $X_{1} \prec_{l r} X_{2}, X_{1} \prec_{h r} X_{2}$ and $X_{1} \prec_{s} X_{2}$.

ii) If $\alpha_{1}=\alpha_{2}, \beta_{1}=\beta_{2}, \theta_{1} \geqslant \theta_{2}$ and $\gamma_{1} \leqslant \gamma_{2}$, then $X_{1} \prec_{l r} X_{2}, X_{1} \prec_{h r} X_{2}$ and $X_{1} \prec_{s} X_{2}$.

\section{Moment}

The $r$ th moment of the EGL distribution can be obtained as

$$
\begin{aligned}
\mu_{r} & =E\left[X^{r}\right] \\
& =\frac{\theta^{\frac{-r}{\beta}} \Gamma\left(\alpha+\frac{r}{\beta}\right)}{\beta(\theta+\gamma) \Gamma(\alpha+1)}\{\alpha \beta \theta+\gamma(\alpha \beta+r)\}, \quad r>-\alpha \beta .
\end{aligned}
$$

Using (12), the expected value is given by

$$
\mu=E[X]=\frac{\theta^{\frac{-1}{\beta}} \Gamma\left(\alpha+\frac{1}{\beta}\right)}{\beta(\theta+\gamma) \Gamma(\alpha+1)}\{\alpha \beta \theta+\gamma(\alpha \beta+1)\} .
$$

\subsection{Moment Generating Function and Characteristic Func- tion}

In the current section, the moment generating and characteristic function of the four parameters EGL distribution are derived.

We know that

$$
M(t)=E[\exp \{t X\}]=\sum_{n=0}^{\infty} \frac{t^{n}}{n !} E\left[X^{n}\right] .
$$

Hence using (12), the moment generating function of the EGL distribution is given by

$$
M(t)=\sum_{n=0}^{\infty} \frac{t^{n} \theta^{\frac{-n}{\beta}} \Gamma\left(\alpha+\frac{n}{\beta}\right)}{n ! \beta(\theta+\gamma) \Gamma(\alpha+1)}\{\alpha \beta \theta+\gamma(\alpha \beta+n)\},
$$


Also the characteristic function of EGL distribution defined by $\phi(t)=$ $E[\exp \{i t X\}]$ takes the form

$$
\phi(t)=\sum_{n=0}^{\infty} \frac{(i t)^{n} \theta^{\frac{-n}{\beta}} \Gamma\left(\alpha+\frac{n}{\beta}\right)}{n ! \beta(\theta+\gamma) \Gamma(\alpha+1)}\{\alpha \beta \theta+\gamma(\alpha \beta+n)\},
$$

where $i=\sqrt{-1}$ is the imaginary number.

\section{Mean Residual Life Function and Scaled Total Time}

In this section, the mean residual life function and scaled total time of the EGL distribution are given.

\subsection{Mean Residual Life Function}

Another important representation for a random variable is the mean residual life (MRL) function defined by

$$
m(t)=E[X-t \mid X>t]=\frac{\int_{t}^{\infty} x f(x) d x}{\bar{F}(t)}-t=\frac{\int_{t}^{\infty} \bar{F}(x) d x}{\bar{F}(t)},
$$

where $\bar{F}(x)=1-F(x)$ is the survival function.

The MRL function as well as failure rate function is very important since each of them can be determined a unique corresponding life time distribution.

Theorem 2. The MRL function of the EGL distribution is

$$
m(t)=\frac{\theta^{\frac{-1}{\beta}}\left\{\alpha \theta \Gamma\left(\alpha+\frac{1}{\beta}, \theta t^{\beta}\right)+\gamma \Gamma\left(\alpha+1+\frac{1}{\beta}, \theta t^{\beta}\right)\right\}}{\alpha \theta \Gamma\left(\alpha, \theta t^{\beta}\right)+\gamma \Gamma\left(\alpha+1, \theta t^{\beta}\right)}-t .
$$

Proof. We have

$$
f(x ; \alpha, \beta, \theta, \gamma)=\frac{\theta}{\theta+\gamma} f_{g g}(x ; \alpha, \beta, \theta)+\frac{\gamma}{\theta+\gamma} f_{g g}(x ; \alpha+1, \beta, \theta),
$$

and thus with some elementary algebraic calculations, the proof is completed. 


\subsection{Scaled Total Time Function}

The scaled total time function is defined by

$$
S_{F}[F(t)]=\frac{1}{\mu} \int_{0}^{t} \bar{F}(u) d u .
$$

If $F(t)$ is the distribution function of the $\operatorname{EGL}(\alpha, \beta, \theta, \gamma)$ distribution, then

$$
\begin{aligned}
S_{F}[F(t)]= & \frac{1}{\mu}\left[\left\{\frac{t}{(\gamma+\theta) \Gamma(\alpha+1)}\right\}\left\{\alpha \theta \Gamma\left(\alpha, \theta t^{\beta}\right)-\gamma \Gamma\left(\alpha+1, \theta t^{\beta}\right)\right\}\right. \\
& \left.-\left\{\frac{(\theta)^{-\frac{1}{\beta}}}{(\gamma+\theta) \Gamma(\alpha+1)}\right\} \Gamma\left(\frac{1}{\beta}+\alpha, \theta t^{\beta}\right)-\gamma \Gamma\left(\frac{1}{\beta}+\alpha+1, \theta t^{\beta}\right)\right],
\end{aligned}
$$

where $\mu$ is obtained from (13).

\section{Lorenz and Bonferroni Curves}

In this section, we give the Lorenz and Bonferroni curves for our proposed distribution.

\subsection{Lorenz Curve}

The Lorenz curve for a positive random variable $X$ is defined as the graph of the ratio

$$
L_{F}(F(y))=\frac{1}{\mu} \int_{0}^{y} u f(u) d u,
$$

against $F(x)$ with the properties $L(p) \leqslant p, L(0)=0$ and $L(1)=1$. If $X$ represents annual income, $L(p)$ is the proportion of total income that accrues to individuals having the $100 p \%$ lowest incomes.

If all individuals earn the same income then $L(p)=p$ for all $p$. The area between the line $L(p)=p$ and the Lorenz curve may be regarded as a measure of inequality of income, or more generally, of the variability of $X$, see Gail and Gastwirth (1978) and Dagum (1985) for extensive discussion of Lorenz curves. 
Theorem 3. The Lorenze curve of EGL distribution be given by

$$
L_{F}(F(y))=1-\frac{\alpha \beta \theta \Gamma\left(\alpha+\frac{1}{\beta}, \theta y^{\beta}\right)+\beta \gamma \Gamma\left(\alpha+\frac{1}{\beta}+1, \theta y^{\beta}\right)}{(\gamma+\alpha \beta \theta+\alpha \beta \gamma) \Gamma\left(\alpha+\frac{1}{\beta}\right)},
$$

Proof. We have

$$
f(x ; \alpha, \beta, \theta, \gamma)=\frac{\theta}{\theta+\gamma} f_{g g}(x ; \alpha, \beta, \theta)+\frac{\gamma}{\theta+\gamma} f_{g g}(x ; \alpha+1, \beta, \theta),
$$

and

$$
\mu=\frac{\theta^{\frac{-1}{\beta}}(\gamma+\alpha \beta \gamma+\alpha \beta \theta) \Gamma\left(\alpha+\frac{1}{\beta}\right)}{\Gamma(\alpha+1) \beta(\theta+\gamma)}
$$

Therefore

$$
\begin{aligned}
L_{F}(F(y))= & \frac{1}{\mu}\left[\frac{\theta}{\theta+\gamma} \theta^{\frac{-1}{\beta}}\left\{\frac{\Gamma\left(\alpha+\frac{1}{\beta}\right)-\Gamma\left(\alpha+\frac{1}{\beta}, \theta y^{\beta}\right)}{\Gamma(\alpha)}\right\}\right. \\
& \left.+\frac{\gamma}{\theta+\gamma} \theta^{\frac{-1}{\beta}}\left\{\frac{\Gamma\left(\alpha+1+\frac{1}{\beta}\right)-\Gamma\left(\alpha+1+\frac{1}{\beta}, \theta y^{\beta}\right)}{\Gamma(\alpha+1)}\right\}\right] \\
= & 1-\left\{\frac{\alpha \beta \theta \Gamma\left(\alpha+\frac{1}{\beta}, \theta y^{\beta}\right)+\beta \gamma \Gamma\left(\alpha+\frac{1}{\beta}+1, \theta y^{\beta}\right)}{(\gamma+\alpha \beta \theta+\alpha \beta \gamma) \Gamma\left(\alpha+\frac{1}{\beta}\right)}\right\} .
\end{aligned}
$$

\subsection{Bonferroni Curve}

The Bonferroni curve has many applications not only in economics to study income and poverty, but also in other fields like reliability, medicine and insurance. The Bonferroni curve $B_{F}[F(y)]$ is given by

$$
B_{F}[F(y)]=\frac{1}{\mu F(y)} \int_{0}^{y} u f(u) d u
$$

Therefore the Bonferroni curve of $F$ that follows the EGL distribution can be obtained via the expression $B_{F}[F(y)]=L_{F}(F(y)) / F(y)$ where

$$
F(y)=\frac{(\gamma+\theta) \Gamma(\alpha+1)-\theta \alpha \Gamma\left(\alpha, \theta y^{\beta}\right)-\gamma \Gamma\left(\alpha+1, \theta y^{\beta}\right)}{(\gamma+\theta) \Gamma(\alpha+1)} .
$$




\section{The Bivariate Case}

In this section, we provide a family of bivariate distributions whose univariate marginals are the extended generalized Lindley distributions. For this, let $\left(V_{1}, V_{2}\right)$ and $\left(W_{1}, W_{2}\right)$ be two vectors of independent random variables distributed according to the $\mathrm{GG}(\alpha, \beta, \theta)$ and $\mathrm{GG}(\alpha+1, \beta, \theta)$, respectively. For $\gamma \geqslant 0$, consider the random pair $\left(X_{1}, X_{2}\right)=\left(V_{1}, V_{2}\right)$ with the probability $\frac{\theta}{\theta+\gamma}$, and $\left(X_{1}, X_{2}\right)=\left(W_{1}, W_{2}\right)$ with the probability $\frac{\gamma}{\theta+\gamma}$. It is then easy to verify that the joint density function of the pair $\left(X_{1}, X_{2}\right)$ is given by:

$$
\begin{aligned}
f\left(x_{1}, x_{2}\right)= & \frac{\theta^{2 \alpha+1} \beta^{2}\left\{\alpha^{2}\left(x_{1} x_{2}\right)^{\alpha \beta-1}+\theta \gamma\left(x_{1} x_{2}\right)^{(\alpha+1) \beta-1}\right\}}{(\theta+\gamma) \Gamma(\alpha+1)^{2}} \\
& \times \exp \left\{-\theta\left(x_{1}^{\beta}+x_{2}^{\beta}\right)\right\},
\end{aligned}
$$

where $x_{1}, x_{2}>0$ and $\alpha, \beta, \theta, \gamma>0$.

Also note that the joint density function (15), may be written as the following model:

$$
f\left(x_{1}, x_{2}\right)=\frac{\theta\left\{\alpha^{2}+\gamma \theta\left(x_{1} x_{2}\right)^{\beta}\right\}}{(\theta+\gamma) \alpha^{2}} f_{g g}\left(x_{1} ; \alpha, \beta, \theta\right) f_{g g}\left(x_{2}, \alpha, \beta, \theta\right) .
$$

When $\gamma=0$, the random variables $X_{1}$ and $X_{2}$ become independent and the bivariate density function (16), reduces to the product of two generalized gamma density functions with the same parameters. The following proposition gives the mixture representation of the conditional density functions of (15).

Theorem 4. If $X_{1}$ and $X_{2}$ are jointly distributed according to (16), then the conditional density function of $X_{j}$ given $X_{i}=x_{i}$; denoted by $f_{j \mid i}\left(x_{j} \mid x_{i}\right)$, is given by:

$$
f_{j \mid i}\left(x_{j} \mid x_{i}\right)=\frac{\alpha}{\alpha+\gamma x_{i}{ }^{\beta}} f_{g g}\left(x_{j}, \alpha, \beta, \theta\right)+\frac{\gamma x_{i}{ }^{\beta}}{\alpha+\gamma x_{i}{ }^{\beta}} f_{g g}\left(x_{j}, \alpha+1, \beta, \theta\right),
$$

Proof. Considering the joint density function of $\left(X_{1}, X_{2}\right)$ in (16) and the marginal density function of $X_{i}, i=1,2$, in (10), using the relation

$$
f_{j \mid i}\left(x_{j} \mid x_{i}\right)=\frac{f\left(x_{i}, x_{j}\right)}{f\left(x_{i}\right)}
$$

the proof is completed. 
Simple calculations show that for each positive integer $m$ and $n$, the following expression for the moments could be obtained

$$
E\left(X_{1}^{n} X_{2}^{m}\right)=\frac{\Gamma\left(\alpha+\frac{n}{\beta}\right) \Gamma\left(\alpha+\frac{m}{\beta}\right)}{\Gamma(\alpha+1)^{2}(\theta+\gamma) \theta^{\frac{m+n}{\beta}}}\left\{\alpha^{2} \theta+\gamma\left(\alpha+\frac{n}{\beta}\right)\left(\alpha+\frac{m}{\beta}\right)\right\} .
$$

A bivariate distribution is said to be positively likelihood ratio dependent (PLRD) if the density function $f(x, y)$ satisfies

$$
f\left(x_{1}, y_{1}\right) f\left(x_{2}, y_{2}\right) \geqslant f\left(x_{1}, y_{2}\right) f\left(x_{2}, y_{1}\right)
$$

for all $x_{1} \geqslant x_{2}$ and $y_{1} \geqslant y_{2}$. For the bivariate density function (16), the above inequality is equivalent to

$$
\left(x_{1}^{\beta}-x_{2}^{\beta}\right)\left(y_{1}^{\beta}-y_{2}^{\beta}\right) \geqslant 0 .
$$

The PLRD has several results in particular, it implies $P\left(X_{1} \leqslant x \mid X_{2}=y\right)$ is non increasing in $y$ for all $x$; and similarly $P\left(X_{2} \leqslant y \mid X_{1}>x\right)$ is non increasing in $x$ for all $y$; This property is called positive regression dependent (PRD).

\section{Different Methods for Estimating}

In this section, maximum likelihood and minimum spacing distance estimators of the parameters of the EGL distribution are discussed.

\subsection{Maximum Likelihood Estimation}

In this part the maximum likelihood estimators of $\operatorname{EGL}(\alpha, \beta, \theta, \gamma)$ are considered. If $X_{1}, \ldots, X_{n}$ is a random sample from $X$ distributed according to EGL, then the log-likelihood function, $l(\alpha, \beta, \theta, \gamma)$ is:

$$
\begin{aligned}
l(\alpha, \beta, \theta, \gamma)= & n(\alpha+1) \log \theta-n \log (\gamma+\theta)-n \log \Gamma(\alpha+1) \\
& +(\alpha \beta-1) \sum \log x_{i}+\sum \log \left(\alpha+\gamma x_{i}{ }^{\beta}\right)-\theta \sum x_{i}{ }^{\beta}+n \log \beta
\end{aligned}
$$


Therefore, the log-likelihood equations are

$$
\begin{aligned}
\frac{\partial l}{\partial \alpha} & =n \log \theta-n \psi(\alpha+1)+\beta \sum \log x_{i}+\sum \frac{1}{\alpha+\gamma x_{i}{ }^{\beta}}=0 \\
\frac{\partial l}{\partial \beta} & =\alpha \sum \log x_{i}+\sum \frac{\gamma\left(\log x_{i}\right) x_{i}{ }^{\beta}}{\alpha+\gamma x_{i}{ }^{\beta}}-\theta \sum x_{i}{ }^{\beta} \log x_{i}+\frac{n}{\beta}=0 \\
\frac{\partial l}{\partial \theta} & =\frac{n(\alpha+1)}{\theta}-\frac{n}{\gamma+\theta}-\sum x_{i}{ }^{\beta}=0 \\
\frac{\partial l}{\partial \gamma} & =\sum_{i=1}^{n} \frac{x_{i}{ }^{\beta}}{\alpha+\gamma x_{i}{ }^{\beta}}-\frac{n}{\gamma+\theta}=0 .
\end{aligned}
$$

And the second derivatives are

$$
\begin{gathered}
\frac{\partial^{2} l}{\partial \alpha^{2}}=-n \psi(\alpha+1)-\sum_{i=1}^{n} \frac{1}{\left(\alpha+\gamma x_{i}^{\beta}\right)^{2}} \\
\frac{\partial^{2} l}{\partial \beta^{2}}=\frac{-n}{\beta^{2}}-\theta \sum\left(\log x_{i} x_{i}^{\beta}\right)^{2}+\sum_{i=1}^{n} \frac{\alpha \gamma x_{i}^{\beta}\left(\log x_{i}\right)^{2}}{\left(\alpha+\gamma x_{i}^{\beta}\right)^{2}}, \\
\frac{\partial^{2} l}{\partial \theta^{2}}=\frac{-n(\alpha+1)}{\theta^{2}}+\frac{n}{(\gamma+\theta)^{2}}, \\
\frac{\partial^{2} l}{\partial \gamma^{2}}=\sum_{i=1}^{n} \frac{x_{i}^{2 \beta}}{\left(\alpha+\gamma x_{i}^{\beta}\right)^{2}}+\frac{n}{(\gamma+\theta)^{2}}
\end{gathered}
$$

where $\psi(\alpha)=\frac{d}{d \alpha} \log \Gamma(\alpha)$ is the digamma function.

The maximum likelihood estimates $\hat{\alpha}, \hat{\beta}, \hat{\theta}$, and $\hat{\gamma}$ for the parameters $\alpha$, $\beta, \theta, \gamma$, respectively, are obtained by solving iteratively Equations (18)-(21).

\subsection{Minimum Spacing Distance Estimator}

In this subsection we provide the minimum spacing distance estimator (MSDE) of the extended generalized Lindley distribution. Let $X_{1}, \ldots, X_{n}$ be a random sample from a continuous population with the $\operatorname{cdf} F_{\boldsymbol{\theta}}, \boldsymbol{\theta} \in \boldsymbol{\Theta} \subset \mathbb{R}^{k}$ with support on $\mathbb{R}$. Let the order statistics be denoted by $Y_{1}, \ldots, Y_{n}$. Define

$$
D_{i}(\boldsymbol{\theta})=F_{\boldsymbol{\theta}}\left(Y_{i}\right)-F_{\boldsymbol{\theta}}\left(Y_{i-1}\right), \quad i=1, \ldots, n+1,
$$


where $F_{\boldsymbol{\theta}}\left(Y_{0}\right)=0$ and $\boldsymbol{F}_{\boldsymbol{\theta}}\left(Y_{n+1}\right)=1$. The MSDE of $\boldsymbol{\theta}$ is obtained by minimizing of

$$
T(\boldsymbol{\theta})=\sum_{i=1}^{n+1} h\left(D_{i}(\boldsymbol{\theta}), \frac{1}{n+1}\right),
$$

over $\Theta$, in which $h(x, y)$ is an appropriate distance. Two choices of $h(x, y)$ are $|x-y|$ and $\log x-\log y \mid$, which are called "absolute" and "absolute log" distance, respectively. These estimators are called "minimum spacing absolute distance estimator" (MSADE) and "minimum spacing absolute log distance estimator" (MSALDE). This estimation method was originally explored by Torabi (2008). We illustrate in the next sections that this method can be used quite successfully for the extended generalized Lindley distribution.

\section{Simulation}

We simulate $n=20,30,50,100$ and 200 times the extended generalized Lindley distribution for $\alpha=6, \beta=0.5, \theta=2.5$ and $\gamma=2.5$. For each sample size, we compute the MLEs, MMEs, MSADEs and MSALDEs of the parameters. We repeat this process 10000 times and compute the average estimate (AE) and MSE. The results are reported in Table 1. The required numerical evaluations were implemented using the $\mathrm{R}$ software through the package (stats4), command mle with the L-BFGS-B method and command nlminb.

Comparing the performance of all estimators, it is observed that for all methods, the MSEs decrease as the sample size increases. Note that, the performances of the MSLDEs are the best as far as the MSE is concerned, but after this method, the MLEs and the MMEs performances are considerable. Considering all the points, we recommend to use the MSALDE for estimating of the parameters. 
Table 1. Estimated AE and MSE of MLE, MME, MSADE and MSALDE of the parameters based on 10000 simulations of the extended generalized Lindley distribution for $\alpha=6, \beta=0.5, \theta=2.5$ and $\gamma=2.5$ and with $n=20,30,50,100$ and 200 .

\begin{tabular}{|c|c|c|c|c|c|c|c|c|c|}
\hline \multirow[b]{2}{*}{$\mathrm{n}$} & & \multicolumn{2}{|c|}{ MLE } & \multicolumn{2}{|c|}{ MME } & \multicolumn{2}{|c|}{ MSADE } & \multicolumn{2}{|c|}{ MSALDE } \\
\hline & & $\mathrm{AE}$ & MSE & $\mathrm{AE}$ & MSE & $\mathrm{AE}$ & MSE & $\mathrm{AE}$ & MSE \\
\hline \multirow[t]{4}{*}{20} & $\alpha$ & 16.139 & 29.516 & 5.772 & 2.367 & 5.775 & 1.091 & 8.172 & 306.74 \\
\hline & $\beta$ & 0.811 & 0.649 & 0.580 & 0.029 & 0.501 & 0.018 & 0.510 & 0.041 \\
\hline & $\theta$ & 10.890 & 23.251 & 2.178 & 0.460 & 2.502 & 0.357 & 4.600 & 216.07 \\
\hline & $\gamma$ & 65.320 & 599.79 & 2.504 & 0.016 & 2.638 & 2.417 & 4.206 & 235.10 \\
\hline \multirow[t]{4}{*}{30} & $\alpha$ & 14.228 & 26.06 & 5.933 & 1.441 & 5.797 & 1.007 & 8.127 & 237.17 \\
\hline & $\beta$ & 0.726 & 0.511 & 0.556 & 0.017 & 0.504 & 0.014 & 0.515 & 0.038 \\
\hline & $\theta$ & 9.365 & 20.63 & 2.282 & 0.270 & 2.472 & 0.322 & 4.462 & 164.95 \\
\hline & $\gamma$ & 42.479 & 491.29 & 2.507 & .009 & 2.609 & 1.346 & 4.685 & 386.83 \\
\hline \multirow[t]{4}{*}{50} & $\alpha$ & 15.061 & 26.040 & 6.009 & 0.613 & 5.829 & 1.402 & 7.291 & 141.68 \\
\hline & $\beta$ & 0.629 & 0.398 & 0.527 & 0.006 & 0.507 & 0.013 & 0.529 & 0.037 \\
\hline & $\theta$ & 9.980 & 20.802 & 2.394 & 0.096 & 2.478 & 0.410 & 3.81 & 97.67 \\
\hline & $\gamma$ & 33.921 & 441.34 & 2.506 & 0.005 & 2.571 & 0.692 & 4.67 & 292.60 \\
\hline \multirow[t]{4}{*}{100} & $\alpha$ & 13.091 & 20.87 & 6.032 & 0.129 & 5.905 & 1.603 & 7.998 & 139.21 \\
\hline & $\beta$ & 0.558 & 0.285 & 0.510 & .001 & 0.505 & .009 & 0.5225 & 0.032 \\
\hline & $\theta$ & 8.210 & 16.47 & 2.465 & 0.010 & 2.511 & 0.509 & 4.279 & 88.95 \\
\hline & $\gamma$ & 3.108 & 27.94 & 2.50 & .001 & 2.69 & 10.03 & 5.517 & 334.61 \\
\hline \multirow[t]{4}{*}{200} & $\alpha$ & 11.800 & 17.46 & 6.007 & 0.073 & 5.999 & 2.431 & 7.067 & 99.54 \\
\hline & $\beta$ & 0.503 & 0.197 & 0.504 & 0.001 & 0.502 & 0.005 & 0.511 & 0.021 \\
\hline & $\theta$ & 7.047 & 12.97 & 2.482 & 0.003 & 2.561 & 1.012 & 3.899 & 64.82 \\
\hline & $\gamma$ & 2.202 & 1.303 & 2.502 & 0.001 & 2.604 & 3.567 & 5.092 & 256.25 \\
\hline
\end{tabular}

\section{Application}

In this section, we use a real data set to show that the extended generalized Lindley distribution can be a better model than the generalized Lindley (GL), power Lindley (PL), Lindley $(\mathrm{L})$, generalized gamma $(\mathrm{GG})$, gamma $(\mathrm{G})$, Weibull (W), exponential (E), Rayleigh (R), half-normal (HN), quasi Lindley (QL), Marshall-Olkin extended Lindley (MOEL) and weighted Lindley (WL) distributions.

In order to compare the models, we used following four criterions: Akaike Information Criterion (AIC), Bayesian Information Criterion (BIC), Consistent Akaike Information Criterion(CAIC), HQIC (Hannan-Quinn informa- 
tion criterion) which are defined as follows:

$$
\begin{gathered}
\mathrm{AIC}=-2 \log \hat{L}+2 k, \quad \mathrm{BIC}=-2 \log \hat{L}+k \log n, \\
\mathrm{CAIC}=\mathrm{AIC}+\frac{2 k(k+1)}{n-k+1}, \quad \mathrm{HQIC}=-2 \log \hat{L}+2 \log \{\log (n)\} k,
\end{gathered}
$$

where $k$ is the number of free parameters in the model and $n$ is the sample size. For fitting a data set, the best model is a model with the smallest value of AIC, BIC, CAIC and HQIC. We can also perform formal goodness-of-fit tests in order to verify which distribution fits better to these data. We apply Kolmogorov-Smirnov (KS) and Anderson-Darling statistics, where small values of thesis statistics for a model indicate that this model could be chosen as the best model to fit the data. The required numerical evaluations were implemented using the $\mathrm{R}$ software through the package (stats4), command mle with the L-BFGS-B method, and package (Adequacy Model), command goodness.fit.

The data set consists of the number of successive failures for the air conditioning system of each member in a fleet of 13 Boeing 720 jet airplanes (1963). The data set is:

194, 413, 90, 74, 55, 23, 97, 50, 359, 50, 130, 487, 57, 102, 15, 14, 10, 57, 320, 261,

$51,44,9,254,493,33,18,209,41,58,60,48,56,87,11,102,12,5,14,14,29,37$,

$186,29,104,7,4,72,270,283,7,61,100,61,502,220,120,141,22,603,35,98$, $54,100,11,181,65,49,12,239,14,18,39,3,12,5,32,9,438,43,134,184,20,386$, $182,71,80,188,230,152,5,36,79,59,33,246,1,79,3,27,201,84,27,156,21,16$, $88,130,14,118,44,15,42,106,46,230,26,59,153,104,20,206,5,66,34,29,26$, $35,5,82,31,118,326,12,54,36,34,18,25,120,31,22,18,216,139,67,310,3,46$, $210,57,76,14,111,97,62,39,30,7,44,11,63,23,22,23,14,18,13,34,16,18,130$, $90,163,208,1,24,70,16,101,52,208,95,62,11,191,14,71$ 
Table 2. MLEs, KS, AD, AICs, BICs, CAICs and HQICs for the real data set.

\begin{tabular}{ccccccccccc}
\hline \hline Dist. & $\hat{\alpha}$ & $\hat{\beta}$ & $\hat{\theta}$ & $\hat{\gamma}$ & $\mathrm{KS}$ & $\mathrm{AD}$ & $\mathrm{AIC}$ & $\mathrm{BIC}$ & CAIC & HQIC \\
\hline EGL & 5.148 & 0.348 & 1.425 & 8.509 & 0.044 & 0.2670 & 2074.1 & 2087.1 & 2074.4 & 2079.9 \\
$\mathrm{GL}$ & $1 \mathrm{e}-09$ & 1 & .0107 & 1.0626 & 0.082 & 1.925 & 2086.3 & 2096.0 & 2086.4 & 2090.2 \\
L & 1 & 1 & 0.0215 & 1 & 0.215 & 23.551 & 2167.3 & 2170.5 & 2167.3 & 2168.6 \\
GG & 0.8083 & 1.04765 & 0.00650 & 0 & 0.086 & 1.861 & 2083.1 & 2089.5 & 2083.1 & 2085.7 \\
G & 0.9048 & 1 & 0.00986 & 0 & 0.069 & 1.286 & 2079.2 & 2085.7 & 2079.3 & 2081.8 \\
E & 1 & 1 & 0.01089 & 0 & 0.084 & 2.032 & 2078.5 & 2081.7 & 2078.5 & 2079.8 \\
W & 1 & 0.91064 & 0.01699 & 0 & 0.057 & 0.947 & 2077.5 & 2084.0 & 2077.6 & 2080.1 \\
PL & 1 & 0.6609 & 0.1088 & 1 & 0.048 & 0.7149 & 2075.4 & 2078.6 & 2075.4 & 2076.7 \\
\hline
\end{tabular}

Table 2 lists the MLEs of the parameters from the fitted models and the values of the following statistics: KS, AD, AIC, BIC, CAIC and HQIC. For Rayleigh distribution, corresponding p-values for the KS and AD statistics are less than 0.05. Based on the values of these statistics, we conclude that the EGL distribution and its sub-models can provide good fits for lifetime data. The E distribution was slightly better based on the values of BIC, HQIC, but the EGL model has the lowest values for the KS, AD, AIC CAIC criteria among the fitted models, and therefore it could be chosen as the best model for these real data.

The plots of the empirical and theoretical density and cumulative distribution function and P-P plot for the EGL and GL distribution are given in Figures 3. This figure shows again that the EGP distribution gives a good fit for these data.

\section{Conclusions}

In this article, we introduced a new flexible extension of the generalized Lindley distribution. We derived some important properties of the new distribution. Using a simulation study, some estimation methods for the parameters of the distribution were compared. Then its application to model a real data set was presented and discussed to demonstrate that this distribution can be used quite effectively to provide better fit than other available subclass models such as the generalized Lindley, power Lindley, Lindley, generalized gamma, gamma, Weibull, exponential, Rayleigh and half-normal, and also some other extensions of the Lindley distribution such as the weighted Lindley and quasi Lindley models. 
Histogram and theoretical densities

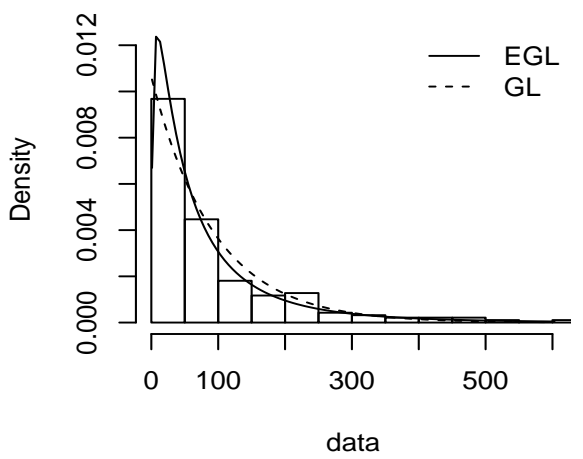

P-P plot

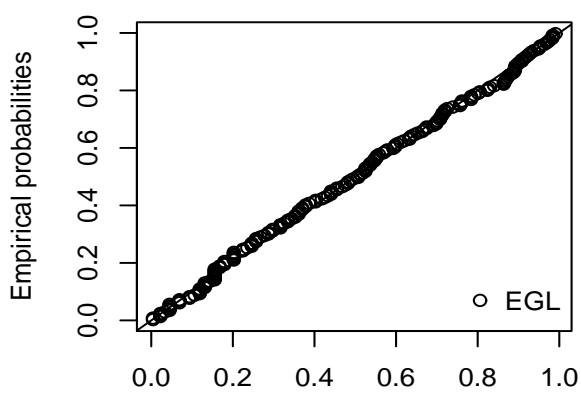

Theoretical probabilities
Empirical and theoretical CDFs

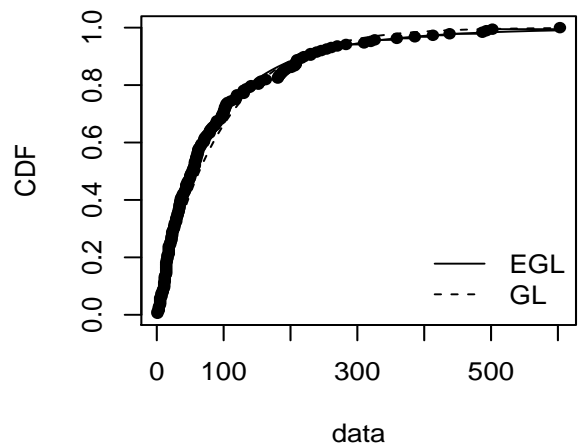

P-P plot

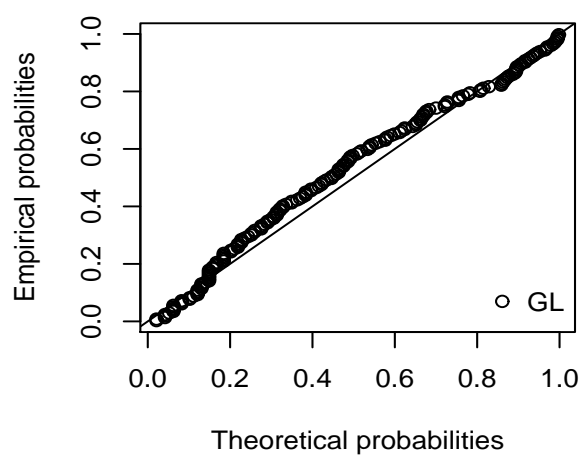

Figure 3. Fitted plots for the EGL and GL distributions.

\section{References}

Dagum, C. (1985). Lorenz Curve, In: S. Kotz, N.L. Johnson, C.B. Read (Eds.), In: Encyclopedia of Statistical Sciences, 5, Wiley, New York, 156-161.

Gail, M.H. and Gastwirth, J.L. (1978). A Scale-free Goodness-of-fit Test for the Exponential Distribution Based on the Lorenz Curve, Journal of the American Statistical Association, 73, 787-793.

Ghitany, M.E., Al-Mutairi, D.K., Al-Awadhi, F.A. and Al-Burais, M.M. (2012). MarshallOlkin Extended Lindley Distribution and Its Application. International Journal of Applied Mathematics, 25, 709-721. 
Ghitany, M.E., Al-Mutairi, D.K., Balakrishnan, N. and Al-Enezi, L.J. (2013). Power Lindley Distribution and Associated Inference. Computational Statistics and Data Analysis, 64, 20-33.

Ghitany, M.E., Alqallaf, F., Al-Mutairi, D.K. and Husain, H.A. (2011). A Two-parameter Weighted Lindley Distribution and Its Applications to Survival Data. Mathematics and Computers in Simulation, 81, 1190-1201.

Ghitany, M.E., Atieh, B. and Nadarajah, S. (2008). Lindley Distribution and Its Applications. Mathematics and Computers in Simulation, 78, 493-506.

Lindley, D.V. (1958). Fiducial Distributions and Bayes' Theorem. Journal of the Royal Society, Series B, 20, 102-107.

Pararai, M., Warahena-Liyanage, G. and Oluyede, B.O. (2015). A New Class of Generalized Power Lindley Distribution with Applications to Lifetime Data, Theoretical Mathematics 86 Applications, 5, 53-96.

Shaked, M. and Shanthikumar, G. (1994). Stochastic Orders. Springer, New York.

Shanker, R. and Mishra, A. (2013). A Quasi Lindley Distribution. African Journal of Mathematics and Computer Science Research, 6, 64-71.

Torabi, H. (2008). A General Method for Estimating and Hypotheses Testing Using Spacings. Journal of Statistical Theory and Applications, 8, 163-168.

Warahena-Liyanage, G. and Pararai, M. (2014). A Generalized Power Lindley Distribution with Applications, Asian Journal of Mathematics and Applications, (Article ID ama0169), $1-23$.

Zakerzadeh, H. and Dolati, A. (2009). Generalized Lindley Distribution. Journal of Mathematical Extension, 3, 13-25.

\section{H. Torabi}

Department of Statistics, Yazd University,

Yazd, Iran.

email: htorabi@yazd.ac.ir

\author{
M. Falahati-Naeini \\ Department of Statistics, \\ Yazd University, \\ Yazd, Iran. \\ email:m.falahati.n@gmail.com
}

\section{N. H. Montazeri}

Department of Statistics,

Yazd University,

Yazd, Iran.

email: nmontazeri@stu.yazd.ac.ir 